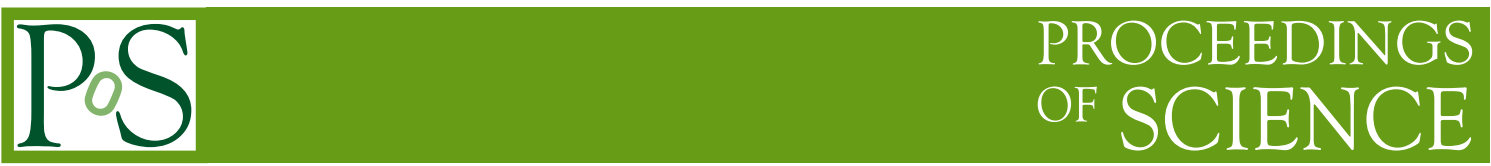

\title{
RX J0818.2+0122 - an AGN with an unusually broad Keplerian rotator line profile
}

\author{
Amogelang M. A. Moeng, ${ }^{a}$ Hartmut Winkler ${ }^{a, *}$ and Francois van Wyk ${ }^{a}$ \\ ${ }^{a}$ Department of Physics, University of Johannesburg, \\ PO Box 524, 2006 Auckland Park, Johannesburg, South Africa
}

E-mail: mogorwama@gmail.com, hwinkler@uj.ac.za, fvw@saao.ac.za

We investigate low resolution spectra and the Balmer line profile in particular of the Active Galactic Nucleus associated with the X-ray source RX J0818.2+0122, and also compare this to an archival Gemini spectrum recorded seven years earlier. This AGN displays broad emission lines with a double bump profile that is characteristic of fast moving gas in a Keplerian orbit around a supermassive black hole. This source is particularly interesting, as the rotational speeds inferred from the line widths reach $15000 \mathrm{~km} \mathrm{~s}^{-1}$, which corresponds to some of the highest values measured in AGN with Keplerian rotator line profiles. We attempt to fit the profile with models developed for this class of objects, and thereby estimate the dimensions, inclination and some additional properties of the broad emission line region of this AGN. Our study highlights a significant weakening as well as considerable profile changes in our latest spectrum. Given that this AGN is also a radio source, we explore the possibility that the peculiar broad line characteristics are related to a radio jet.

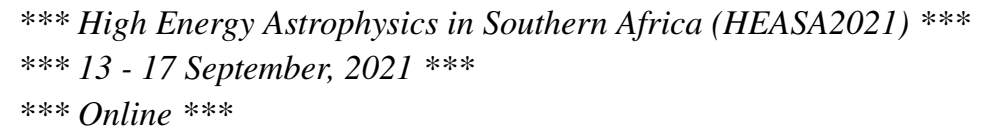

${ }^{*}$ Speaker 


\section{Introduction}

RX J0818.2+0122 was identified with the galaxy LEDA $1192920(\mathrm{RA}(2000)=08 \mathrm{~h} 18 \mathrm{~m} 14.7 \mathrm{~s}$, $\left.\operatorname{Dec}(2000)=+01^{\circ} 22^{\prime} 27^{\prime \prime}\right)$ and discovered to be a Seyfert galaxy during optical follow-up observations of new X-ray sources detected by the ROSAT mission [1]. A radio image was secured soon after discovery with a faint hint of a jet [2]. $1.4 \mathrm{GHz}$ radio emission was measured to be $21.3 \mathrm{mJy}$. This object has exceptionally broad Balmer lines which also display emission peaks well to the blue and red of the central wavelength, something curiously not mentioned in previous papers that published data on this target. Such profiles are typically associated with emission line generating gas in orbital motion about a central black hole. The mathematical formulation for such a physical scenario were developed into a series of Keplerian rotator line profile models by Chen, Halpern, Eracleous and collaborators [3-5]. Whenever the broad $\mathrm{H} \alpha$ line's blue and red edges stretch beyond both the [O I] $6300 \AA$ and [S II] $6725 \AA$ lines respectively these correspond to exceptionally high rotational speeds of at least $12000 \mathrm{~km} \mathrm{~s}^{-1}$. Speeds of this magnitudes and similarly distorted line profiles are only encountered rarely. Examples of Seyfert galaxies with such properties include 1E 0450.3-1817 [6], 3C 332 [5] and Pictor A [7].

\section{Observation and data reduction}

We obtained a low resolution spectrum of RX J0818.2+0122 on 2 April 2019 with the SpUpNIC spectrograph [8] mounted on the $1.9 \mathrm{~m}$ telescope at the Sutherland site of the South African Astronomical Observatory (SAAO), with integration times of $2 \times 1200 \mathrm{~s}$. We used a slit width translating to 2.7 arcsec on the sky, and positioned the grating angle so that it covered the wavelength range 3300-8100 $\AA$. An argon spectrum was obtained to perform the wavelength calibration immediately before and after each AGN spectrum. After applying routine data reduction procedures (bias subtraction, flatfield correction, cosmic ray removal, wavelength and flux calibration) the spectrum was corrected for interstellar reddening applying the extinction values at those galactic coordinates determined by Schlafly and Finkbeiner [9].

One earlier spectrum of RX J0818.2+0122 has been published, recorded with the Gemini-North telescope on 18 March 2012 [10] and available online in a fully reduced form. While this spectrum only ranged from 4700-7650 $\AA$, it had very low noise levels and includes both $\mathrm{H}_{\alpha}$ and $\mathrm{H}_{\beta}$. The same interstellar reddening correction was applied here as for the 2019 spectrum.

\section{Spectral analysis}

To initiate the spectral analysis we first transformed the wavelengths to the rest frame defined here by cross-correlating our spectrum with a Seyfert 1 galaxy template of [11]. This immediately yields the target redshift of $z=0.891$.

A substantial fraction of the measured spectrum is made up of normal starlight from the host galaxy, far from the active nucleus, but that cannot in practice be excluded by collimation. We therefore specifically assume that the spectral form of this component corresponds to that of regular non-active galaxies. The strength of this component can be estimated by identifying standard absorption features in the combined spectrum and compare those to a host galaxy template. For 


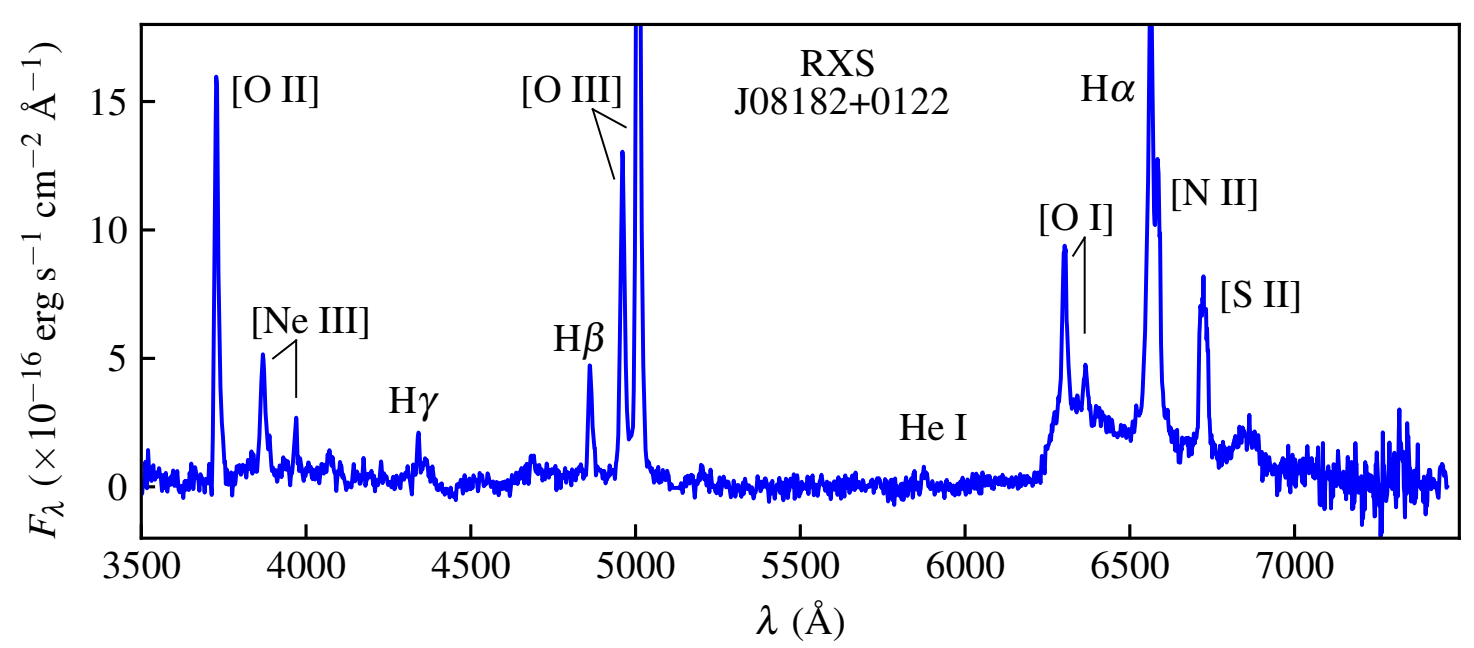

Figure 1: Rest frame SAAO Spectrum of RX J0818.2+0122, with host galaxy and power law components subtracted.

this we used the "Sb type" galaxy template $\Gamma_{\lambda}$ of [12] (scaled so that its value is 1 at $5100 \AA$ ) and subtracted this from our combined spectrum, applying a scaling factor $g$ that best cancelled out the normal galaxy absorption features in the observed spectrum.

The nuclear spectrum consists of a continuum component that can often be adequately represented by a power law and an emission line spectrum that is in turn subdivided into broad ("b") and narrow ("n") components. So the total spectral flux is

$$
F_{\lambda, \text { tot }}=F_{\lambda, \mathrm{b}}+F_{\lambda, \mathrm{n}}+\left(p(\lambda / 5100)^{\alpha}+g \Gamma_{\lambda}\right) F_{5100}
$$

where $\lambda$ must be in $\AA$ ngstrom and $F_{5100}=6.07 \times 10^{-16} \mathrm{erg} \mathrm{s}^{-1} \mathrm{~cm}^{-2} \AA^{-1}(=0.527 \mathrm{mJy})$ is the spectral flux we measured at $5100 \AA$. The 2019 rest frame spectrum after subtraction of the host galaxy and power law components is shown in Fig. 1. This corresponds to the following parameters: $\alpha=-0.3$, $p=0.38$ and $g=0.62$. We estimate the uncertainties in these parameters to be $\Delta \alpha= \pm 0.3$, $\Delta p= \pm 0.05$ and $\Delta g= \pm 0.62$.

Before fitting Keplerian rotator models, we subtracted the narrow lines from the Balmer broadline profiles. The strengths of most of the narrow emission lines have been compiled in Table 1 . Only the stronger member of the forbidden line doublets are listed, as the flux ratio between these and the weaker member are fixed at $R(6300 / 6363)=3.027$ for [O I] and $R(6584) /(6548)=3.054$ for [N II] [13]. With the narrow line contributions accounted for, it is possible to measure the relative $\mathrm{H}_{\alpha}$ and $\mathrm{H}_{\beta}$ broad line fluxes. Relative to [O III] $5007 \AA$ these fluxes amounted to the following in 2012 and 2019 respectively: $R(H \alpha / 5007)_{2012}=2.66 ; R(H \beta / 5007)_{2012}=0.64$; $R(H \alpha / 5007)_{2019}=1.65 ; R(H \beta / 5007)_{2019}=0.30$.

The residual broad line profiles can now be compared to each other (see Fig. 2). The red function constitutes our attempt to match the observed profiles with Keplerian rotator models [3] [4]. This succeeds reasonably well for the 2019 spectrum despite a moderate overestimation at the red end. The fit is however clearly unsatisfactory for the 2012 spectrum, where there is a significant 


\begin{tabular}{c|cccccccc} 
Line & {$[\mathrm{O} \mathrm{II}]$} & {$[\mathrm{Ne} \mathrm{III}]$} & $\mathrm{H} \gamma$ & $\mathrm{He} \mathrm{II}$ & $\mathrm{H} \beta$ & {$[\mathrm{O} \mathrm{I}]$} & $\mathrm{H} \alpha+[\mathrm{N} \mathrm{II}]$ & {$[\mathrm{S} \mathrm{II}]$} \\
$\lambda(\AA)$ & 3727 & 3869 & 4340 & 4686 & 4861 & 6300 & 6563 & 6725 \\
\hline$R(\lambda / 5007)_{2012}$ & - & - & 0.027 & 0.019 & 0.064 & 0.236 & 0.647 & 0.245 \\
$R(\lambda / 5007)_{2019}$ & 0.373 & 0.137 & 0.046 & 0.018 & 0.099 & 0.223 & 1.002 & 0.304
\end{tabular}

Table 1: $R(\lambda / 5007)_{\text {year }}$ represents the narrow emission line fluxes relative to [O III] $5007 \AA$. The table only lists the stronger transition of the forbidden line pairs, as these doublets have fixed flux ratios [13].
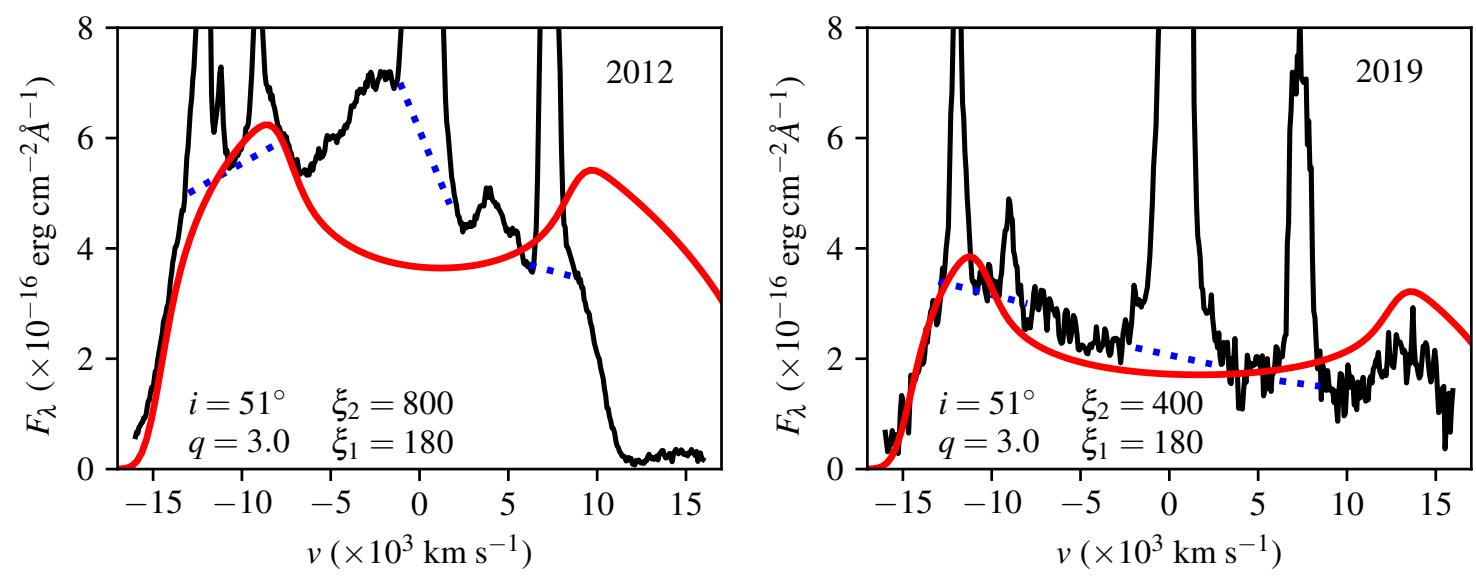

Figure 2: The red line displays the attempted Keplerian rotator model fits to the $\mathrm{H}_{\alpha}$ profiles of 2012 and 2021. The blue dotted lines approximate the divides between the broad component and the superimposed narrow lines.

central enhancement and the red wing has been effectively truncated.

An integration of the line flux over a line's entire wavelength range allows the determination of the $\mathrm{H}_{\alpha}$-to- $\mathrm{H}_{\beta}$ ratio and the measurement of a $\sim 50$ per cent decrease in $\mathrm{H}_{\alpha}$ between 2012 and 2019. We also highlight other significant changes in the 2019 broad line profile compared to seven years earlier: i) the red edge at $\sim 11000 \mathrm{~km} \mathrm{~s}^{-1}$ now extended to significantly larger values $\left(\sim 16000 \mathrm{~km} \mathrm{~s}^{-1}\right)$; ii) the emission bumps visible in the 2012 spectrum between -6000 and $0 \mathrm{~km} \mathrm{~s}^{-1}$ and near $+4000 \mathrm{~km} \mathrm{~s}^{-1}$ had effectively disappeared.

\begin{tabular}{clcc} 
Parameter & Decscription & 2012 & 2019 \\
\hline$i$ & Keplerian disk angle of inclination & $51^{\circ}$ & $51^{\circ}$ \\
$\xi_{1}$ & inner Keplerian disk radius, in units of $G M / c^{2}$ & 100 & 100 \\
$\xi_{2}$ & outer Keplerian disk radius, in units of $G M / c^{2}$ & 800 & 400 \\
$q$ & disk emissivity power law index & 3.0 & 3.0 \\
$\sigma$ & broadening parameter & 2.4 & 2.4
\end{tabular}

Table 2: Keplerian rotator model parameters (see [3, 4]).

We next explore the possibility that part or all of the broad line component is due to outflowing gas rather than being entirely the product of Keplerian rotation. In the Introduction we already alluded to traces of a jet in radio images. Furthermore, our spectral decomposition identified a 


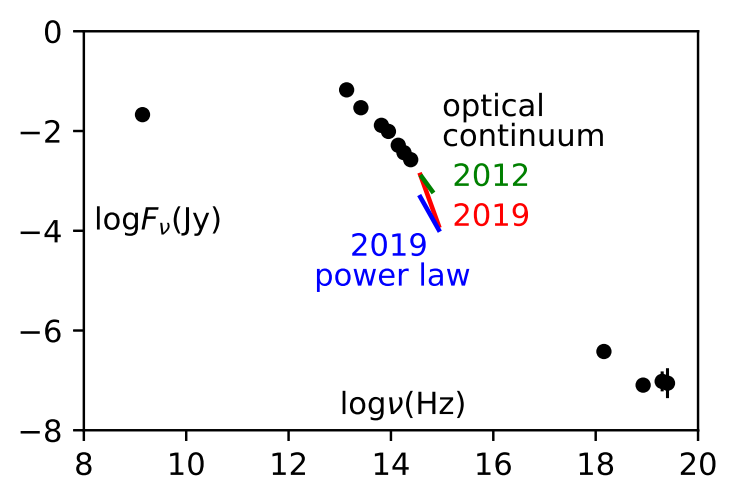

Figure 3: Radio-to-X-ray spectral flux distribution of RX J0818.2+0122. The optical continuum is displayed in green for 2012 and red for 2019, while the 2019 power law component is shown in blue. The radio, infrared and X-ray points are from the NASA Extragalactic Database.

power law component (see Equation 1) that could be due to synchrotron emission. Converting the power law contribution and index to a frequency spectrum results in the optical bandwidth segment illustrated in Fig. 3. This plot furthermore shows flux measurements in other wavebands recorded in the NASA Extragalactic Database. This indicates that the optical measurements are consistent with values in the infrared and X-ray regimes, both in terms of power law index as well as size.

\section{Discussion and Conclusion}

The $\mathrm{H}_{\alpha}$ profile of RXJ0818.2+0122 is largely consistent with that of a Keplerian rotator accretion disk. At the red end of the profile the measured flux is however weaker than that projected by the model. This is particularly true for the 2012 spectrum, where no emission is seen from gas moving away from us at faster than $11000 \mathrm{~km} \mathrm{~s}^{-1}$ compared to the system rest frame. This behaviour might be explained if that part of the emission line region is heavily obscured by line-of-sight dust.

The form of flux distribution from the radio to the X-ray regime is consistent with a synchrotron spectrum (e.g. [14]), which is typically linked to a jet, though the latter cannot be proven as no radio images have been published since the inconclusive original ones in [2]. This opens the possibility that some of the emission contributing to the Balmer line profile is from ejected gas clouds along the jet. How likely would it be that the observed profile is the result of bipolar outflow along the jet axis rather than due do Keplerian rotation? This would arise if the brightest emitting regions are also moving away from the nucleus the fastest. However, the problem with a jet-induced profile is that such dynamics are expected to rather lead to logarithmic profiles [15], which are not consistent with the multiple bump structure of the broad line shapes. We thus conclude that the dominant mechanism generating the unusual broad line profiles in RX J0818.2+0122 is due to Keplerian rotation, though more complex models still need to be developed to properly account for the observed spectra. 


\section{Acknowledgements}

The authors gratefully acknowledge the allocation of South African Astronomical Observatory telescope time. This research has made use of the NASA/IPAC Extragalactic Database (NED), which is funded by the National Aeronautics and Space Administration and operated by the California Institute of Technology.

\section{References}

[1] N. Bade, D. Engels, W. Voges, V. Beckmann, T. Boller, L. Cordis et al., The Hamburg/RASS Catalogue of optical identifications, Astron. Astrophys. Suppl. 127 (1998) 145.

[2] F.E. Bauer, J.J. Condon, T.X. Thuan and J.J. Broderick, RBSC-NVSS Sample. I. Radio and Optical Identifications of a Complete Sample of 1556 Bright X-Ray Sources, Astrophys. J. Suppl. 129 (2000) 547.

[3] K. Chen, J.P. Halpern and A.V. Filippenko, Kinematic Evidence for a Relativistic Keplerian Disk: ARP 102B, Astrophys. J. 339 (1989) 742.

[4] K. Chen and J.P. Halpern, Structure of Line-emitting Accretion Disks in Active Galactic Nuclei: ARP 102B, Astrophys. J. 344 (1989) 115.

[5] M. Eracleous, M. Livio, J.P. Halpern and T. Storchi-Bergmann, Elliptical Accretion Disks in Active Galactic Nuclei, Astrophys. J. 438 (1995) 610.

[6] S.A. Stephens, Optical Spectroscopy of X-Ray-Selected Active Galactic Nuclei, Astron. J. 97 (1989) 10.

[7] M. Eracleous and J.P. Halpern, Accurate Redshifts and Classifications for 110 Radio-Loud Active Galactic Nuclei, Astrophys. J. Suppl. 150 (2004) 181.

[8] L.A. Crause, D. Gilbank, C.v. Gend, H.L. Worters, C. Sass, E.J. Kotze et al., SpUpNIC (Spectrograph Upgrade: Newly Improved Cassegrain): a versatile and efficient low- to medium-resolution, long-slit spectrograph on the South African Astronomical Observatory's 1.9-m telescope, J. Astron. Telesc. Instrum. Syst. 5 (2019) 024007.

[9] E.F. Schlafly and D.P. Finkbeiner, Measuring Reddening with Sloan Digital Sky Survey Stellar Spectra and Recalibrating SFD, Astrophys. J. 737 (2011) 103.

[10] M. Koss, B. Trakhtenbrot, C. Ricci, I. Lamperti, K. Oh, S. Berney et al., BAT AGN Spectroscopic Survey. I. Spectral Measurements, Derived Quantities, and AGN Demographics, Astrophys. J. 850 (2017) 74.

[11] N. Pol and Y. Wadadekar, Seyfert 1 composite spectrum using SDSS Legacy survey data, Mon. Not. R. Astron. Soc. 465 (2017) 95 [1610.07523]. 
[12] A.L. Kinney, D. Calzetti, R.C. Bohlin, K. McQuade, T. Storchi-Bergmann and H.R. Schmitt, Template Ultraviolet to Near-Infrared Spectra of Star-forming Galaxies and Their Application to K-Corrections, Astrophys. J. 467 (1996) 38.

[13] P.J. Storey and C.J. Zeippen, Theoretical values for the [OIII] $5007 / 4959$ line-intensity ratio and homologous cases, Mon. Not. R. Astron. Soc. 312 (2000) 813.

[14] M. Böttcher, A. Reimer and A.P. Marscher, Implications of the very High Energy Gamma-Ray Detection of the Quasar 3C279, Astrophys. J. 703 (2009) 1168.

[15] W. Kollatschny and M. Zetzl, The shape of broad-line profiles in active galactic nuclei, Astron. Astrophys. 549 (2013) A100 [1211. 3065]. 